\title{
Enterovesical Fistula and Bladder Calculus caused by a Migrated Intrauterine
}

\section{Device: A Case Report}

\section{Niurka Wallace $^{1 *} \mid$ Shenelle Wilson ${ }^{2}$}

*Correspondence: Niurka Wallace

Address: ${ }^{1}$ Rowan University School of Osteopathic Medicine, Medical Center Drive, Stratford NJ; ${ }^{2}$ Georgia Urology, Atlanta, Georgia

e-mail $\bowtie:$ wallac13@rowan.edu

Received: 13 September 2021; Accepted: 21 September 2021

Copyright: (C) 2021 Wallace N. This is an open-access article distributed under the terms of the Creative Commons Attribution License, which permits unrestricted use, distribution, and reproduction in any medium, provided that the original work is properly cited.

\begin{abstract}
Intrauterine devices (IUD) have proven to be one of the most reliable methods of contraception. However, as it is a foreign body, it can migrate from the intended position in the uterus, perforating surrounding structures and creating fistulas. We report a case of a 42-year-old woman who presented with abdominal pain, recurrent urinary tract infections, and chronic pelvic pain for eight years. Imaging revealed an intravesical IUD with attached bladder calculus, and a transurethral cystolitholapaxy subsequently uncovered an enterovesical fistula. Conservative urethral catheter management did not resolve the fistula, which was then repaired via robotic excision. The patient had an uncomplicated postoperative course, and voiding cystourethrogram confirmed water-tight repair of the fistula tract after two weeks.
\end{abstract}

Keywords: Intrauterine Device, Enterovesical Fistula, Bladder Calculi, Chronic Pelvic Pain

\section{Introduction}

The intrauterine device (IUD) is a reversible, economical, highly effective contraceptive, with one of the lowest failure rates and the highest continuation rates in the general population (Krashin et al., 2015). However, IUDs can migrate, causing severe but rare complications days to years after insertion, such as uterine perforation, colonic perforation, intestinal obstruction, and fistula formation (Almarhabi, 2020). These complications are often unnoticed as they carry a low index of suspicion, thus making diagnosis difficult. Additionally, some patients experience nonspecific inflammatory symptoms such as cystitis, frequent urination, urinary urgency, dysuria, and discomfort of the lower abdomen, thus further causing a delay in the relevant examination, diagnosis, and treatment (Wan et al., 2021). Another rare complication of IUD migration is the formation of vesical calculus or bladder stone. Bladder calculi are usually a manifestation of underlying pathology, including urinary stasis, infection, urinary tract obstruction, voiding dysfunction, or foreign body retention (Schwartz et al., 2000). The foreign body can be selfinflicted or iatrogenic. It may enter the bladder from the genitourinary tract, bowel, or perivesical tissues. Pelvic ultrasonography and plain X-ray are diagnostic of bladder stones and radio-opaque foreign body 
and can be used for early detection (Abdulwahab-Ahmed and Ogunleye, 2013). We report the case of a woman found to have an enterovesical fistula, an abnormal connection between the bladder and the intestine caused by a migrating IUD, as well as a bladder stone.

\section{Case Presentation}

Our patient is a 42-year-old woman who presented with an 8-year history of vague abdominal discomfort exacerbated by physical activity, chronic pelvic pain, and recurrent urinary tract infections (UTIs). She had a history of multiple copper IUDs placed for contraception in her home country Eritrea in 2012. After the first IUD was inserted, she developed intractable abdominal pain that lasted a few weeks. When she returned to her gynecologist three weeks after IUD placement, she reports being told that the IUD had fallen out because the string was not visible on pelvic exam. A second IUD was placed at that time without incident or post-procedure pain. Over the next few years, she reports intermittent, vague lower abdominal pain that resolved with rest. In 2019, the second IUD was removed, causing subsequent worsening persistent abdominal pain. In December 2020, the patient underwent computerized tomography (CT) scan, which showed the first IUD in the bladder with a $12 \times 12 \mathrm{~mm}$ stone attached (Fig. 1) and a left pelvic kidney. In January 2021, the IUD and stone were removed via a transurethral cystolitholapaxy, revealing a vesical fistula where the IUD was attached (Fig. 2). A catheter was left in place for passive fistula closure. However, a retrograde cystogram performed after one month demonstrated a persistent fistula to the small bowel (Fig. 3). The patient also reported seeing food particles within her catheter bag.

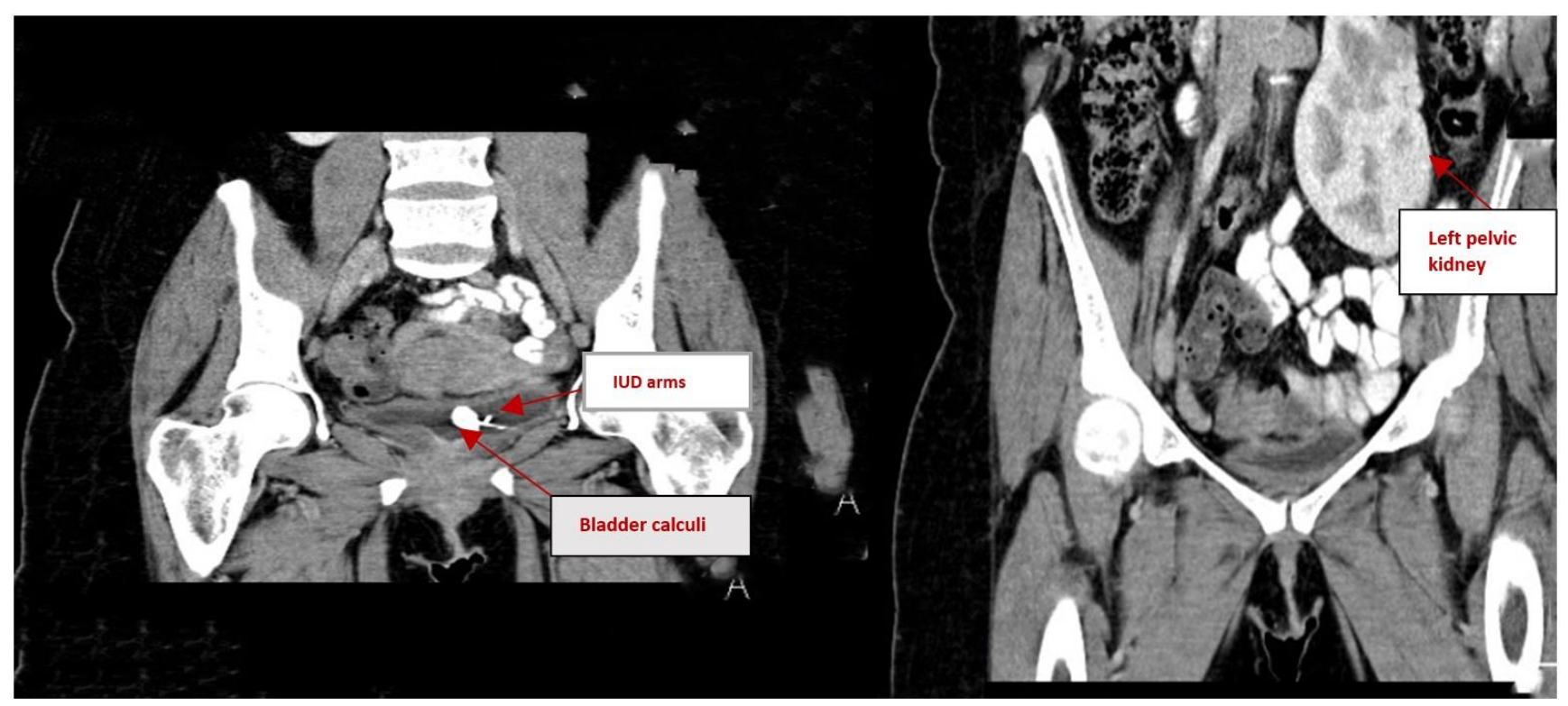

Figure 1: CT images showing IUD and bladder calculus. 


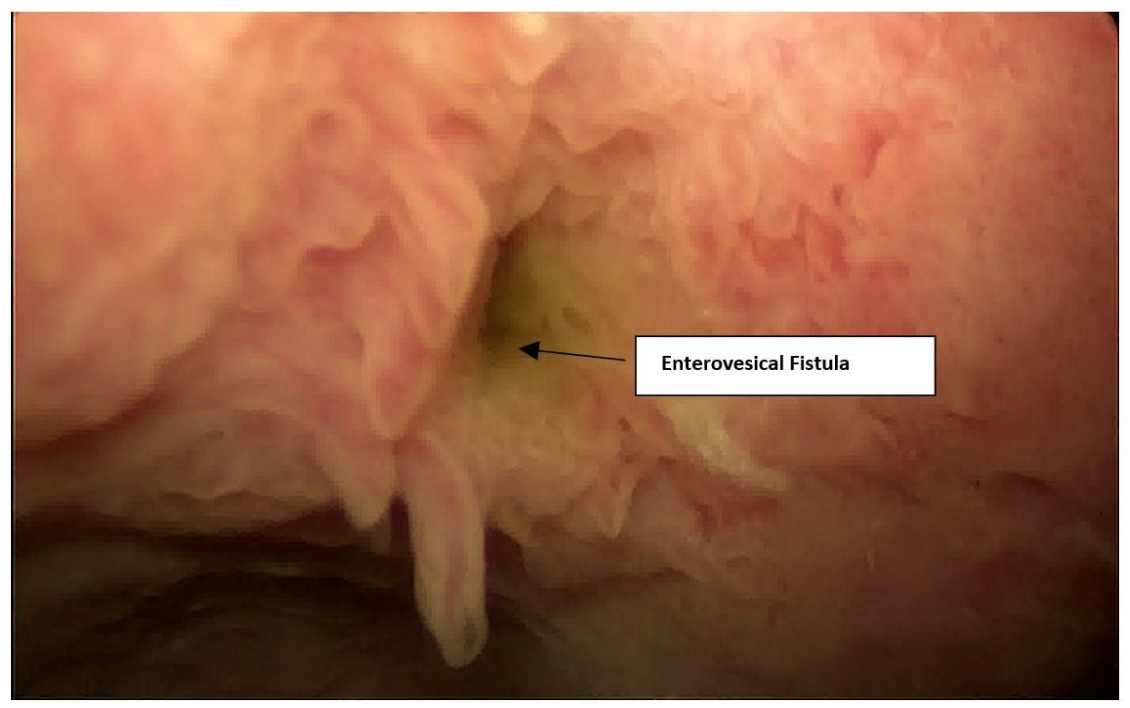

Figure 2: Vesical fistula visualized after removal of IUD and attached bladder stone.

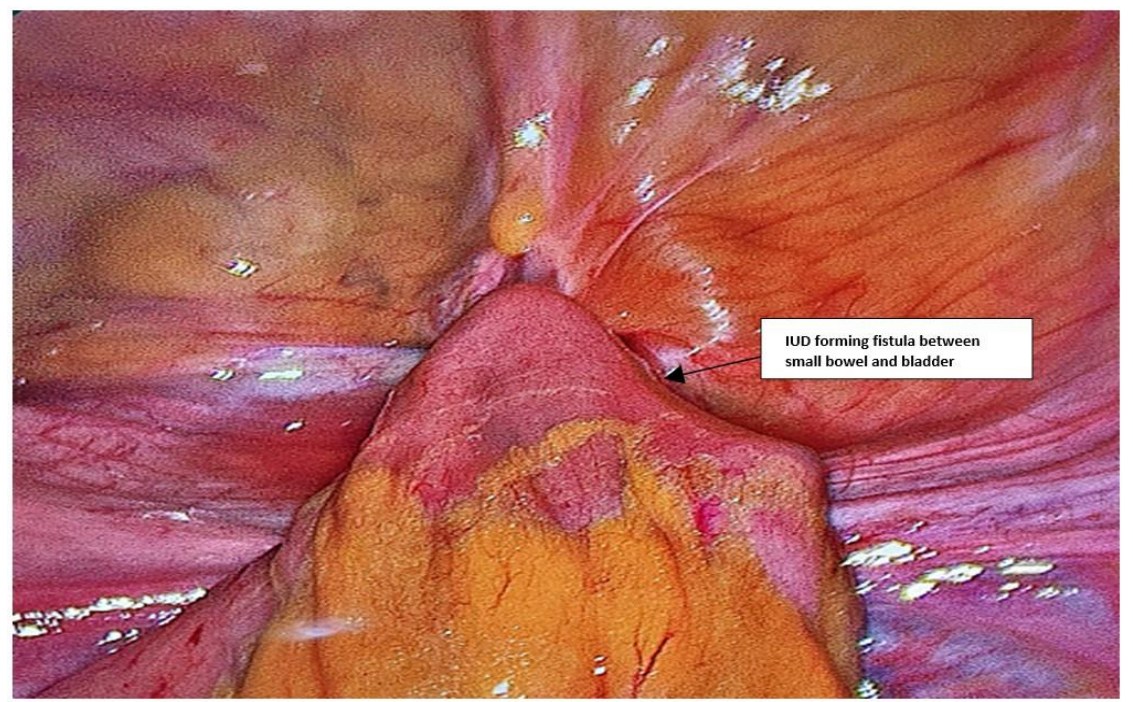

Figure 3: Enterovesical fistula visualized one month post transurethral cystolitholapaxy.

The patient was taken for definitive surgical treatment. She underwent cystoscopy with stent placement within the fistulous tract, then robotic enterovesical fistula excision and small bowel resection. The patient was discharged four days later after the resolution of postoperative ileus. Voiding cystourethrogram then confirmed a water-tight repair at the two-week postoperative visit, and the patient reported resolution of all pre-and peri-operative symptoms after six weeks.

\section{Discussion}

Migration of IUD into surrounding organs is a rare but severe complication after uterine perforation (Cheung et al., 2018). It is often asymptomatic but may be associated with vague pelvic or abdominal pain (Almarhabi, 2020). 
Likely migration sites include the bladder, rectosigmoid colon, peritoneum, appendix, small bowel, and adnexa (Cheung et al., 2018). Once the IUD penetrates the uterus and bladder wall, it can act as a nidus for calculus formation. However, the incidence of an IUD migration to the bladder and stone formation is about 1 per 1000 insertions (Cheung et al., 2018), making this a rare complication of IUD placements. Our patient had an extremely rare case as the IUD migrated to the bladder forming a stone and one arm of the device created a fistula between the bladder and small bowel.

When an IUD string is not visualized on a gynecological examination, this should prompt further detailed investigation. Diagnostic methods include urine examination, abdominal ultrasound, a transvaginal ultrasound, KUB x-ray, and pelvic CT or MRI (Chai et al., 2017). However, CT is most useful for identifying the exact location of the IUD and diagnosing whether it is penetrating surrounding organs (Madden et al., 2016). If the IUD migrates intraperitoneally, minimally invasive techniques or an open approach may be used to solve any associated complications as well as to remove the device, whereas, in incidents of intravesical translocation and stone formation, cystolitholopaxy of the bladder calculus should be employed along with the removal of the IUD (Madden et al., 2016).

This case presentation is unique for several reasons. First, our patient reported an atypical finding of increased abdominal pain after the removal of the visualized IUD. Second, unlike prior published case reports, the second IUD migrated to our patient's bladder, creating a vesical fistula and a subsequent enterovesical fistula. Finally, the closure of the first fistula tract was complicated by food particles repeatedly clogging our patient's foley because of the additional perplexing enterovesical fistula. Due to the lack of similar reported cases seen in urogynecology practice, there is no consensus on surgical approach and management. We hope that this case report will serve as a reference when cases of similar complexity are encountered.

\section{Conclusion}

Intrauterine devices are one of the safest and most reliable methods of contraception. However, like all foreign objects placed in the body, the IUD is not without complications. Although severe complications are rare, they may include bladder calculi, fistula formation between the bladder, and small bowel, all of which were seen in this patient. Fortunately, our patient had a positive outcome and has not reported any complications since her last follow-up.

\section{Abbreviations}

IUD: Intrauterine device

CT: Computed Tomography Scan

UTI: Urinary Tract Infection 


\section{References}

Abdulwahab-Ahmed A and Ogunleye 00. Vesical calculus 10 years post missing intrauterine contraceptive device. J Surg Tech Case Rep 2013; 5: 48-50.

Almarhabi Y. Asymptomatic cecal perforation and ileocecal fistula after intrauterine device migration: a case report. J Surg Case Reports 2020; 2020: 1-3.

Chai W, Zhang W, Jia G, Cui M, Cui L. Vesical transmigration of an intrauterine contraceptive device. Medicine (United States) 2017; 96: 4-6.

Cheung ML, Rezai S, Jackman JM, Patel ND, Bernaba BZ, Hakimian O, Nuritdinova D, Turley CL, Mercado R, Takeshige T, Reddy SM. Retained Intrauterine Device (IUD): Triple Case Report and Review of the Literature. Case Rep Obstet Gynecol 2018; 2018: 1-8.

Krashin J, Tang JH, Mody S, Lopez LM. Hormonal and intrauterine methods for contraception for women aged 25 years and younger. Cochrane Database Syst Rev 2015; (8).

Madden A, Aslam A, Nusrat NB. A Case of Migrating "Saf-T-Coil” Presenting With a Vesicovaginal Fistula and Vesicovaginal Calculus. Urol Case Reports 2016; 7: 17-19.

Schwartz BF, Stoller ML. The vesical calculus. Urol Clin North Am 2000; 27: 333-346.

Wan L, Wang Y, Xiao C, Li X, Cao J, Wang S, Wei X, Liu X. Four cases of heterotopia of an intrauterine device embedded in the bladder muscular layer causing cystolithiasis: case report and review of the literature. J Int Med Res 2021; 49: 300060520979444. 